\title{
MÉTODO CIENTÍFICO E INTERFERENCIAS COMERCIALES EN LA CONSTRUCCIÓN DE LA CIENCIA DEL PROTOCOLO. UNA REFLEXIÓN CRÍTICA PARA UN PROCESO INNOVADOR Y CREÍBLE
}

Fernando Ramos Fernández': Universidad de Vigo. España. ferramos@telefonica.net

\section{RESUMEN}

La construcción de una teoría científica del Protocolo; es decir, elevar el Protocolo a la categoría de Ciencia social, dentro de las de la Comunicación, ha de pasar por una rigurosa serie de fases mediante el método científico, delimitando el objeto de estudio, una vez depurado de las excrecencias comerciales que hoy en día la perturban. El marco donde se produzca esa transformación debe ser la Universidad, a través de la investigación, la publicación y el contraste de los datos obtenidos, en la convicción de que el proceso será largo, que exigirá paciencia y rigor y que no existen atajos. En este artículo estudiamos hasta qué punto, se están planteando adecuadamente los elementos necesarios para que el Protocolo se convierta en una ciencia y no meramente en una técnica auxiliar dentro de la política de imagen de las instituciones públicas y privadas como lo es actualmente. Ello va a requerir profundos cambios y superar los viejos criterios que convertían al Protocolo en una mera técnica de ordenación de las autoridades o los dirigentes de una institución, en función de su rango, en un acto público.

PALABRAS ClAVE: Ciencia - Método - Divulgación - Contraste - Teorías Protocolo.

\section{SCIENTIFIC METHOD AND COMMERCIAL INTERFERENCES IN}

\footnotetext{
${ }^{1}$ Autor correspondiente:

Fernando Ramos Fernández: Profesor Titular de Deontología y Derecho de la Información y la Publicidad en la Universidad de Vigo. España.

Correo: ferramos@telefonica.net
} 


\title{
THE CONSTRUCTION OF THE SCIENCE OF THE PROTOCOL. A CRITICAL REFLECTION FOR AN INNOVATIVE AND CREDIBLE PROCESS
}

\begin{abstract}
The construction of a scientific theory of the Protocol; to raise the Protocol to the category of social Science, inside the set of the Communication, it has to happen for a rigorous series of phases by means of the scientific method, delimiting the object of study, once purified of the commercial excrescences that today bother her. The frame where this transformation happens must be the University, across the investigation, the publication and the contrast of the obtained information, in the conviction that the process will be long, that will demand the patience and the rigor and that do not exist short-cuts. In this article we study the necessary elements in order that the Protocol turns into a science and not simply in an auxiliary technology inside the politics of image of public of institutions like it it is nowadays. This is going to need serious changes and to conquer the old criteria of that Protocol in a mere technology of order of the authorities or the leaders of an institution, depending his range, in a public act.
\end{abstract}

KEY WORDS: Science - Method - Spreading - Contrast - Theories - Protocol.

\section{INTRODUCCIÓN}

Desde que la Doctora María Teresa Otero Alvarado² presentó su tesis doctoral en la Universidad de Sevilla, pionera en plantear el estudio del Protocolo y el Ceremonial como una materia científica "per se", dentro de la gran familia de los Estudios de Comunicación, se marcó un camino para que los interesados en el conocimiento científico -y no meramente descriptivo o casuístico- del Protocolo se adentraran con la adecuada metodología y herramientas en este escasamente hollado territorio. Pero la tesis de la Doctora Otero no sólo incluye su experiencia como jefe de Días Nacionales en la Exposición Universal de Sevilla de 1992. Penetra en la Historia, el Derecho, la Sociología y la Comunicación de la historia de la Civilización en su conjunto, marcando "los tempos" secuenciados que marcan la antigua y moderna presencia del Protocolo como referente esencial en aquélla. Por eso es científica. Dicho de otro modo más sencillo, si queremos desarrollar una Ciencia del Protocolo no basta con describir el "Qué", sino explicar el "Por qué y el Cómo".

El problema que ahora afrontamos es, hasta qué punto, se están planteando adecuadamente los elementos necesarios para que el Protocolo se convierta en una ciencia y no meramente en una técnica auxiliar dentro de la política de imagen de las instituciones públicas y privadas. El saber científico ha de ser objetivo, racional, sistemático, general, falible y metódico. Una vez definido el fenómeno - objeto de

2 Vid. OTERO ALVARADO, $\mathrm{M}^{\mathrm{a}}$ Teresa (1999). Protocolo y relaciones públicas de Estado. Los días nacionales en la exposición Universal de Sevilla. Tesis Doctoral. Universidad de Sevilla. Facultad de Comunicación. 
estudio-, busca conocer las causas y las leyes que lo rigen (Babini, 1975). El Protocolo es una ciencia de resultados que se expresa, de manera muy diversa, según las distintas culturas, pero que coinciden en el concepto de la "intuición de lo correcto"; es decir, del comportamiento humano adecuado y tempestivo en determinados actos y acontecimientos de la vida social, especialmente de aquellos que tienen un carácter simbólico, ritual o conmemorativo, de modo especialmente intenso en el mundo oficial.

Todo el mundo tiene sentido de lo correcto, como todo el mundo lo tiene de lo jurídico, expresado en el lenguaje común ("No hay derecho, tengo derecho, es mi derecho"). Lo mismo ocurre con otro concepto social definible según la cultura de cada momento, el honor, lo honorable. Nos preguntamos si podemos considerar que el Protocolo sea, pueda llegar o no llegar nunca a ser una ciencia en el sentido de "todo conocimiento de la realidad en sus causas, y dotado de un instrumento específico, el método, que le permite alcanzar sus fines", (Carreras Panchón, 1994). Desde una interpretación restrictiva, no pasaría de ser una técnica, pero sin los requisitos para ser considerado propiamente una ciencia. Para saberlo, hemos de fijarnos si, por sus propios contenidos, el Protocolo permite desarrollar una teoría científica a partir de los postulados ordinarios del trabajo intelectual.

En estos momentos, el Protocolo está de moda, y existe una sobreoferta -en gran medida comercial, y de calidades diversas- de formación en este sentido. La variedad oscila desde las materias de Protocolo en otras carreras matrices de Comunicación, especialmente en la de Publicidad y Relaciones Públicas, a diversos grados de variadísimo nombre, aunque todos sean esencialmente lo mismo, con el Protocolo como eje central, preferentemente en universidades privadas y alguna pública, a diversos institutos y escuelas privados con toda clase de ofertas, convenios con universidades, pasarelas y otros cursos, además de los más diversos post grados oficiales y títulos propios por toda la geografía nacional. Es casi imposible el recuento.

Aparecen excesivos intereses de orden puramente comercial (muy respetables en una economía de mercado) que han encontrado en la formación en protocolo un verdadero filón, hasta el punto de haber diseñado un sistema para la captación de alumnosclientes que, con escaso equipaje de origen, lleguen a la misma meta (por la vía de las convalidaciones y pasarelas) que quienes han de adquirir para este viaje billete completo. No deja de ser curioso que, desde alguna de estas plataformas, se pretenda haber descubierto la piedra filosofal y se otorguen la capacidad exclusiva de construir una ciencia del protocolo como si cuestión tan delicada admitiese atajos y mezclan lo que puede llegar a ser una disciplina científica en un mero breviario de tópicos barnizados con apariencia de saber.

\subsection{Estado de la cuestión.}


La Ciencia avanza a través de la investigación ordenada, ya que permite una reconstrucción conceptual de la realidad, que es cada vez más amplia, profunda y exacta. Aborda problemas reales y trata de descomponerlos, de entender sus componentes; intenta descubrir los elementos que componen cada totalidad, y las interconexiones que explican su integración. Hemos de ubicar necesariamente el Protocolo en el territorio de las llamadas "Ciencias Formales" que, como su nombre lo indica, estudian las formas. Dado que el Protocolo se instala dentro de los objetivos de comunicación de las entidades, las empresas y las instituciones, donde, a nuestro entender, se abre un campo inmenso de posibilidades para convertirlo en una disciplina plenamente científica, con elementos propios, que permita formular una teoría diferenciada y que lo rescate del rango de técnica auxiliar al servicio de la imagen de las instituciones. Los hechos del Protocolo son los acontecimientos sociales de cierto rango, no los hechos naturales; pero que están sometidos a unas determinadas reglas, usos y tradiciones formales, expresados a través de la etiqueta y el ceremonial, muchas veces sustentado en la tradición y en los usos sociales consolidados. A lo largo de estos siglos hasta nuestros días, la investigación histórica mostró que el Protocolo y el Ceremonial formaban parte de las preocupaciones prioritarias del Poder, de modo que aparecen sucesivos documentos que perfilan hasta extremos increíbles la vida de la Corte y las relaciones entre la sociedad y el que la gobierna.

Como es sabido, dos centros, unos privado y otro público, se apresuraron a ofrecer,

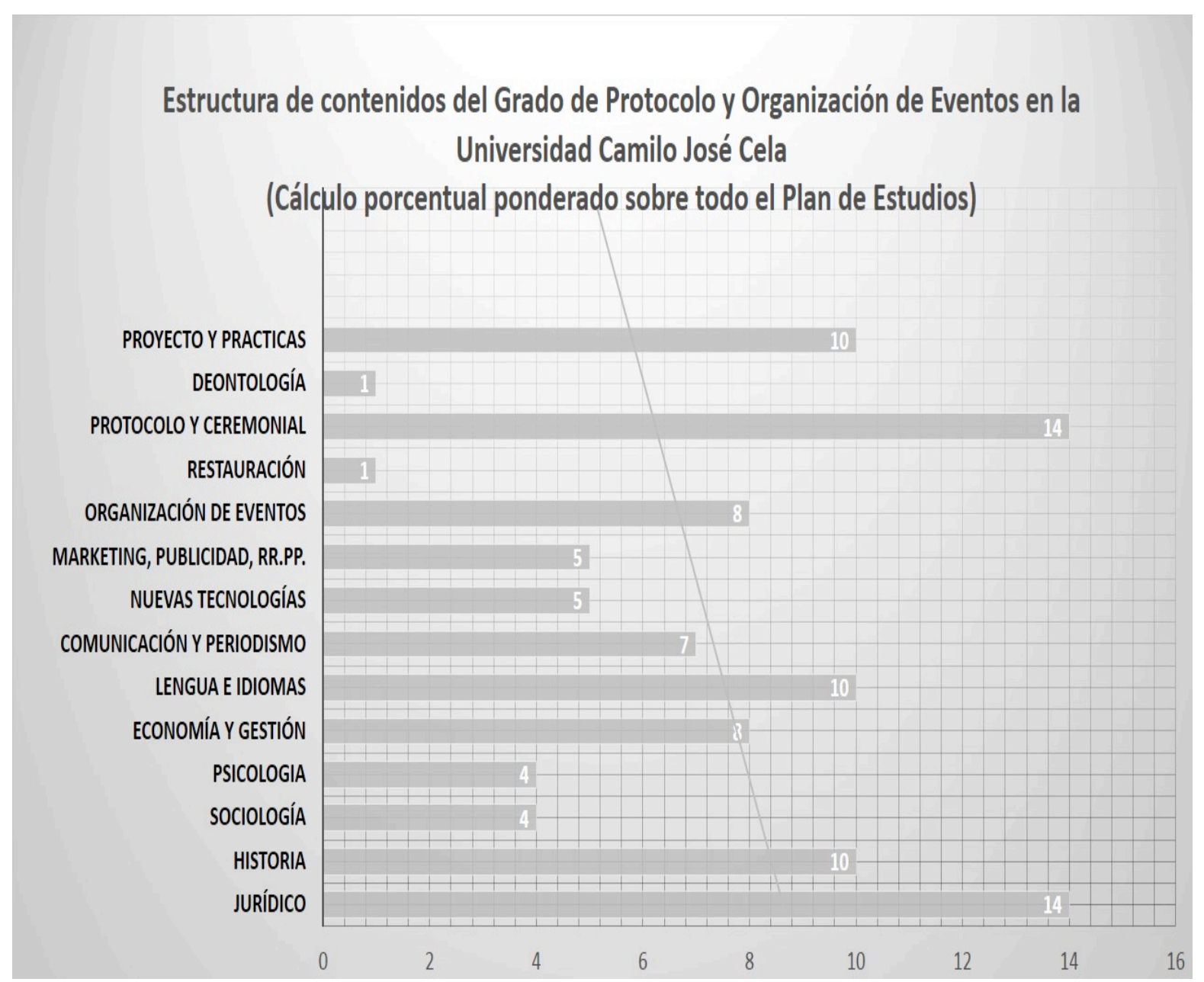


previa sanción por la ANECA -de modo inusualmente rápido- el Grado de Protocolo, con perfiles muy parecidos de estructura y contenido, siendo lo más sorprendente, empero, que entre las salidas que ofertan o prometen a sus egresados sean las mismas que ya cubren otras más tradicionales carreras de comunicación, especialmente las de Relaciones Públicas y también periodismo. No obstante lo dicho, cabe observar que en el diseño de los curricula de estas carreras, por lo que se refiere a las asignaturas básicas $\mathrm{u}$ obligatorias, tienen especial peso, calculado porcentualmente sobre el conjunto de los contenidos, las materias jurídicas, las de la familia de la comunicación en general, aparte de las más específicas de protocolo.

\section{GRADO DE ORGANIZACIÓN DE EVENTOS, PROTOCOLO Y RELACIONES INSTUCIONALES UNIVERSIDAD MIGUEL HERNÁNDEZ}

(CÁLCULO PORCENTUAL DE CONTENIDOS SOBRE EL PLAN DE ESTUDIOS)

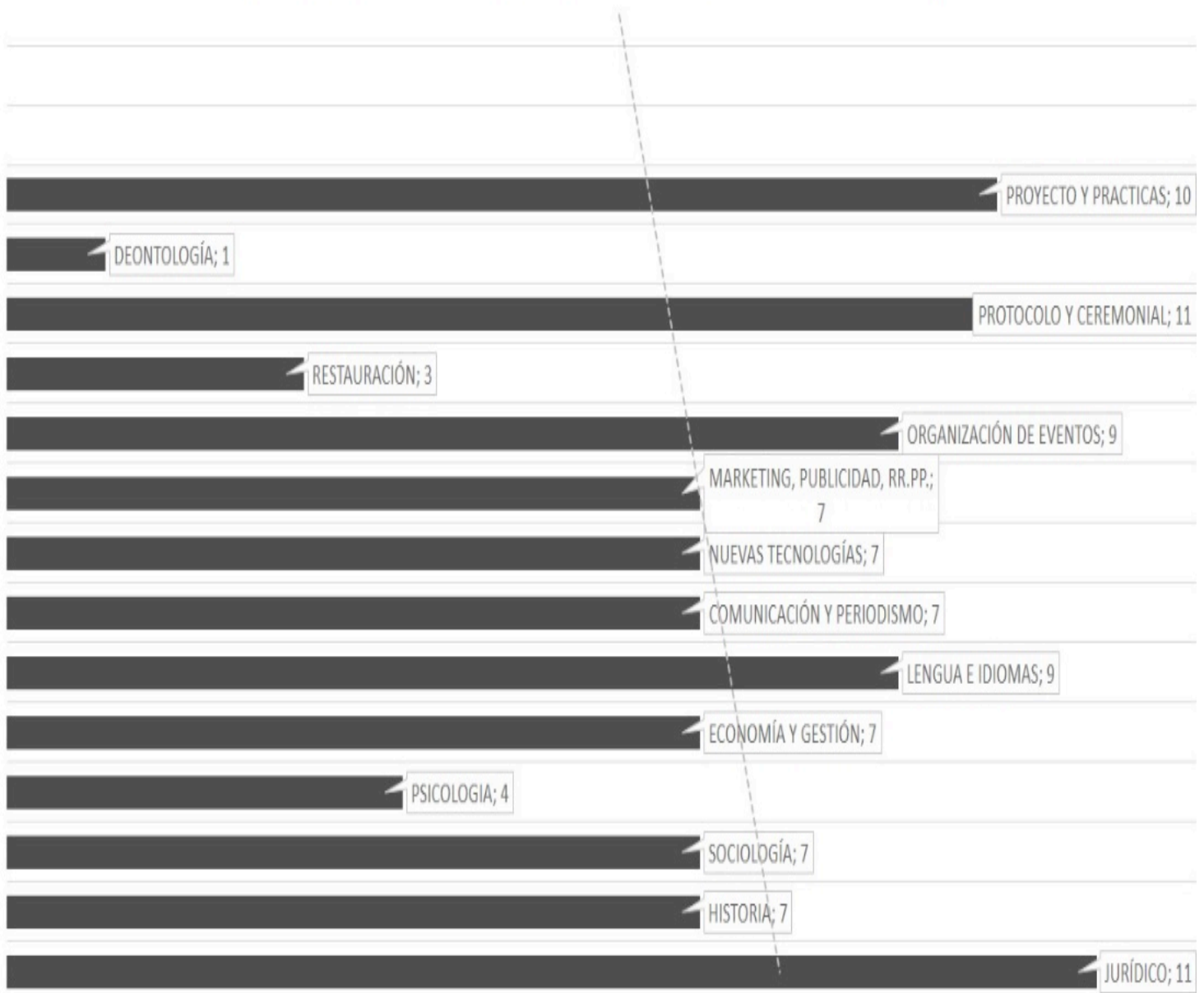

Es curioso observar que en ambos casos, los dos centros coinciden en el volumen de contenidos jurídicos y de protocolo, así como en otros contenidos. En algunos casos se presta mayor atención a las nuevas tecnologías, pero desde una perspectiva de conjunto, los diseños de la carrera es muy parecido, si bien la Universidad Miguel Hernández pone especial énfasis en las materias de sociología y psicología. 


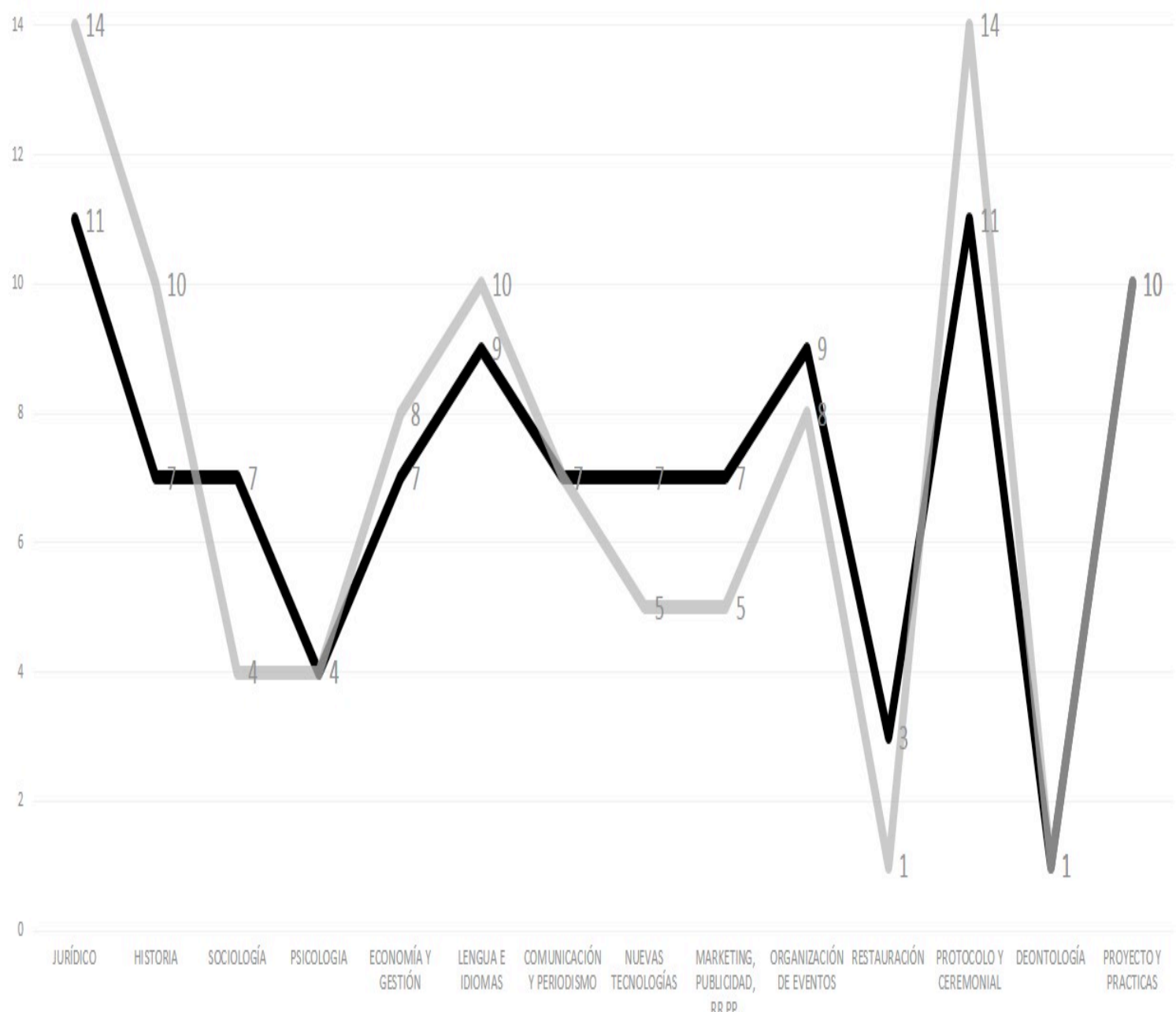




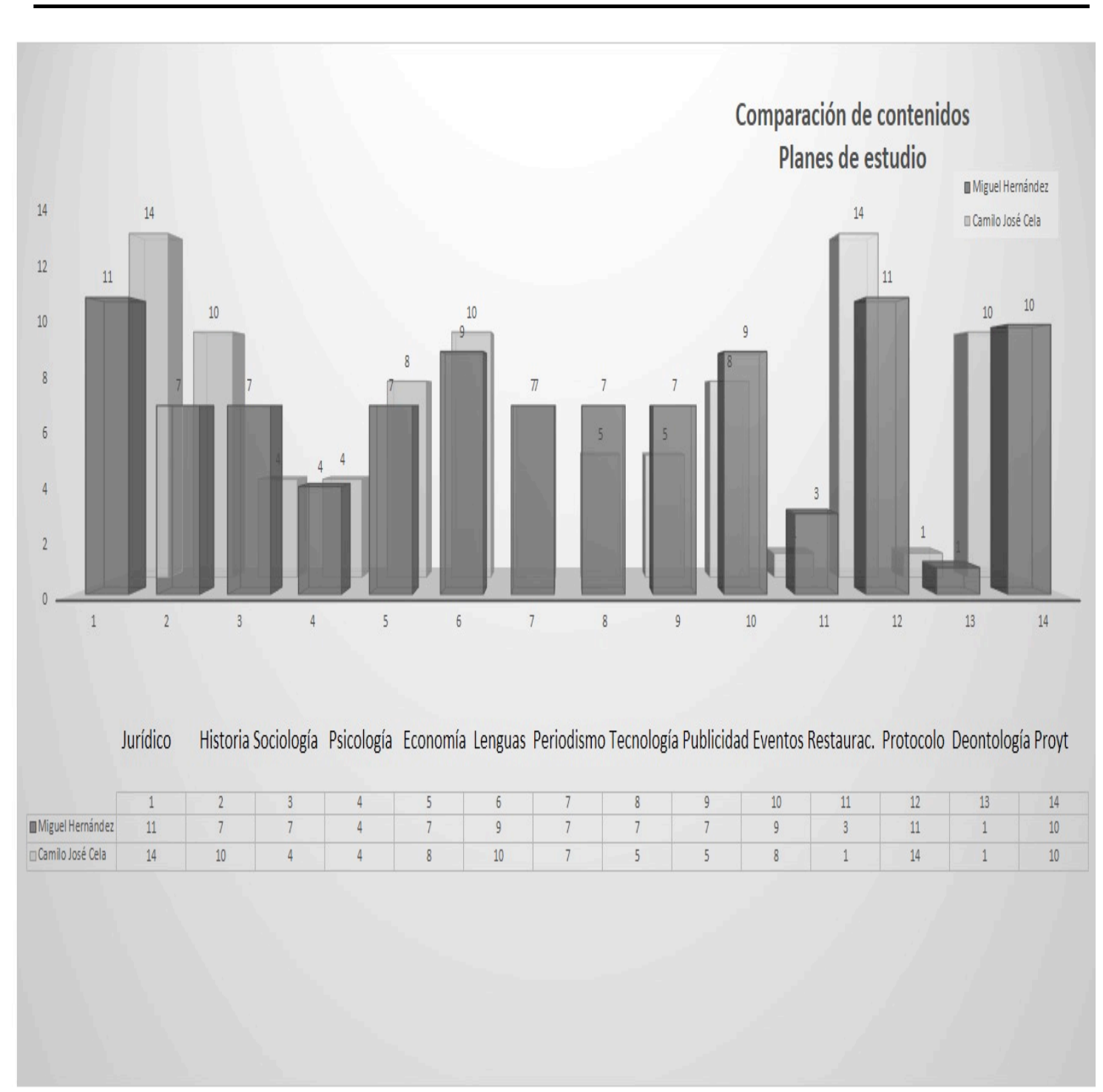

\section{METODOLOGÍA}

Cohen y Nagel (1973: 23) determinan que el "método científico es la persistente aplicación de la lógica para poner a prueba nuestras impresiones, opiniones y conjeturas, examinando las mejores evidencias disponibles a favor y en contra de ellas". Es un modo de resolver problemas siguiendo una forma de actuación que consiste, esencialmente, en observar, clasificar, demostrar e interpretar fenómenos, de manera que se posibilite la predicción y la explicación de cuestiones significativas (González, 1977: 13). Ander-Egg (1996: 24) afirma que el saber científico se obtiene mediante procedimientos metódicos con pretensión de validez, utilizando la reflexión sistemática, los razonamientos lógicos y respondiendo a una búsqueda intencionada. Además, añade que:

Para la búsqueda se delimita el problema que se estudia, se diseña la investigación, se prevén medios e instrumentos de indagación y se procede a un análisis e interpretación 
de todo lo estudiado. En suma: el conocimiento científico es el resultado de una tarea de investigación que se vale del método científico ${ }^{3}$.

Por lo tanto, siguiendo esta pauta, para determinar si es susceptible de ser considerada como tal, y una vez admitido que puede llegar a ser una ciencia, alcanzar ese objetivo, hemos de seguir un método pautado, en las siguientes estaciones.

a) Marco Epistemológico. Que os acerque al conocimiento del por qué.

b) Marco Metodológico: El método cualitativo que incluye todas aquellas investigaciones orientadas hacia la solución de problemas y toma de decisiones vinculadas a la investigación-acción.

c) Marco Conceptual. Se tratan los antecedentes históricos de la materia y se fijan los contenidos.

d) Marco Temporal. Debe ser acotado con precisión. Podemos estudiar la Historia toda la Humanidad, o los siglos actuales.

e) Marco Espacial. Europa, España, el Mundo...

A la hora de establecer el marco científico para elevar el Protocolo a la categoría de Ciencia que merezca ese nombre, hemos de situarnos con todo rigor ante la forma en que se genera el conocimiento y cómo se confronta y santifica; es decir, su epistemología. Su función es analizar los conceptos que se emplean para justificar los datos científicos, considerando los factores sociales, psicológicos y hasta históricos que entran en juego. Dicho de otro modo, nos planteamos las preguntas y tratamos de hallar las respuestas a través de la reflexión razonada. Luego, hemos de comprobar si el resultado es inteligible para nosotros mismos y para quienes estén dispuestos a que lo trasmitamos.

No menos relevante será el método y la precisión conceptual, por cuanto será las herramientas efectivas para avanzar en el proceso trazado. No podremos comprender el sentido simbólico del acto de juramento de un Rey si no acudimos a los antecedentes históricos desde que los germanos elevaban a sus monarcas en un escudo como señal de que estaban por encima de los demás o los rituales de unción sagrada o los símbolos del poder que llegan a nuestros días y conservan singular vigencia, como el hecho mismo de que el acto jurídico-administrativo de toma de posesión de un alcalde se perfecciona cuando éste recibe el bastón de mando. Lo mismo cabe decir de las ceremonias de presentación de cartas credenciales, en las que el embajador pasa a serlo (tras el previo reconocimiento del "placet"), cuando entrega al jefe del Estado ante el que representa al suyo las "Cartas credenciales" y éstas son recibidas como tal, momento en que públicamente es reconocido como embajador.

En cuanto a los marcos temporal y espacial, quedan determinados por el propio significado de ambos términos. En nuestro caso, hemos de recopilar el conocimiento a través de la observación directa de la serie de fenómenos que se suceden en torno al protocolo como herramienta al servicio de la construcción de un determinado mensaje, 
y en cuya génesis convergen elementos jurídico-formales, sociológicos, escénicos y arquitectónicos, verbales y no verbales, entre otros.

Nuestro objetivo es determinar si el Protocolo es una ciencia o si puede llegar a serlo. Nuestro acercamiento a ese problema sigue dos direcciones: el diacrónico y el sincrónico. Contemplamos la historia de la Humanidad en su conjunto, y nos adentramos, esencialmente, en ambos casos en el conjunto de reglas (jurídicas y morales o tabúes en las sociedades más primitivas) que la han gobernado; en las diversas manifestaciones de costumbres y usos sociales en las diversas culturas; analizamos las coincidencias y divergencias. Vemos qué hay de común en determinados ritos, asunción de roles, manifestación de la autoridad y el poder. La consulta de las fuentes del Protocolo nos lleva esencialmente en dos direcciones: La formulación jurídica del protocolo oficial, que los estados expresan a través de leyes y/o reglamentos específicos, por parte de las autoridades públicas, desde el Estado a los municipios, y los manuales de identidad corporativa, libros de estilo o normas de protocolo corporativo, del que se dotan las grandes empresas e instituciones privadas. Pero todas, sean oficiales o no, son normas dictadas para ser cumplidas por quien debe hacerlo.

\section{ANÁLISIS Y DISCUSIÓN}

La expresión hipótesis para referirnos a cualquier enunciado que está sometido contrastación, con independencia de si se propone describir algún hecho o evento concreto, expresar una ley general, o alguna otra proposición más compleja (Hempel, 1983: 38). Además de delimitar el objeto sobre el que elaboramos nuestro estudio, elegimos los objetivos que queremos alcanzar al final del camino, el enfoque empleado, y el desarrollo del trabajo. Una vez delimitado el problema a investigar, definido por una pregunta surgida de una observación más o menos estructurada, el paso siguiente es preguntarse cuál o cuáles son las soluciones más probables para resolverlo. Es decir, determinamos la parcela de la realidad que queremos trabajar, y en ella aparecerá una serie de interrogantes que constituyen la hipótesis de partida.

La investigación científica, en su sentido más amplio, presenta dos vertientes distintas: el descubrimiento de los hechos y la creación de hipótesis y teorías. En el ámbito de las Ciencias Sociales, "las hipótesis son tentativas de explicación de los hechos y fenómenos a estudiar que se formulan al comienzo de una investigación, mediante una suposición o conjetura verosimil, destinada a ser probada por la comprobación de los hechos" (Alcina, 1999: 71).

Si a lo largo de la historia hallamos la presencia del "Protocolo y el Ceremonial", en diversas formas, en todos los pueblos y culturas que alcanzaron los más elevados grados de civilización, parece evidente que este fenómeno no puede quedar reducido al relato puramente mecánico de ritos y ceremonias, ya que se nos abre para profundizar en el estudio más profundo de lo que ha significado y significa desde el punto de vista histórico, político, sociológico y humano. Es evidente que para analizar científicamente y explicarse qué es el Protocolo y para formular una teoría sobre su conocimiento es imprescindible dotarse de otros recursos que la mera revisión de la 
amplísima bibliografía descriptiva (manuales, prontuarios, legislación, libros de estilo, tratados de etiqueta y ceremonial que hallamos en el mercado de las ideas).

La Historia, el Derecho (y en especial alguna de sus ramas o especialidades, desde el Derecho Diplomático al Derecho Premial), la Heráldica, la Vexicología, la Sociología, la Etnografía, la Retórica, la Literatura, la Proxemia, el Paralenguaje, la Kinesia y hasta la Mitología, entre otros, serán conocimientos que es preciso explorar para estructurar el armazón del conocimiento científico del Protocolo. Para formular una teoría general del Protocolo es preciso superar la manida concepción que lo sitúa como una regla de comportamiento humano vinculada al ceremonial y a la etiqueta de determinadas ceremonias palatinas y oficiales. Hemos de entenderlo, por extensión, como el modo correcto de llevar a cabo y ejecutar una acción humana con éxito dentro de sus propios objetivos. De este modo hablamos del protocolo de los quirófanos, del protocolo de la emergencia en un submarino nuclear, etc. El término Protocolo tiene, pues, en nuestros días, un sentido polisémico. Es decir, con el mismo se pueden definir varios casos diferentes. Pero en sentido estricto, como gusta en definirlo la doctora Otero se refiere exclusivamente a la representación pública de la estructura del poder.

HP1. El Protocolo como ciencia es un agregado de conocimientos, desde la sociología al lenguaje verbal y no verbal, que adquiere forma jurídica a través de la norma que establece una determinada pauta ritual y determina la relación espacio temporal de los sujetos que actúan en la vida pública y el mundo social. Su síntesis, en cuanto a la construcción de una teoría científica, obliga a resguardarse bajo una serie de ciencias matrices, desde el Derecho a la Historia, le Heráldica, la Sociología, los lenguajes simbólicos, la vexicología y otra serie de ciencias menores.

\subsection{La Teoría del Honor Social.}

Pierre de Fouquières en el prólogo del célebre tratado de Protocolo francés de Serres señala que las reglas y costumbres que han contribuido a que Francia sea reconocida por sus modales y su elegancia deben servir de ejemplo a todos aquellos que quieran desarrollar con dignidad y eficacia sus funciones públicas (Sarres, Jean, 1982: 8 y ss. Prólogo de Fouquière), en tanto Sgrselli indica que la expresión "Protocolo" expresa siempre un orden y una fórmula honorífica que debe ser respetada. (Sgrelli, 2006: 24 y ss). "El Protocolo es una forma de honor social", Weber (1922: 45, 683 y ss.). En su célebre compendio de saberes "Economía y Sociedad"4 nos enseña que la vida cotidiana, tanto en el pasado como en el presente, las normas morales son, en oposición a las normas jurídicas, considerando la cosa sociológicamente, normas de conducta condicionadas por la religión o por la convención; y sus límites, con respecto al derecho, son graduales.

Como es sabido, en el Protocolo y el ceremonial hallamos normas jurídicas, morales y sociales. Pero ese conjunto de reglas, deberes morales y usos y costumbres son la urdimbre que, entrelazada construye el protocolo. Pero como sabemos, en nuestra

\footnotetext{
4 Seguimos la décima reimpresión para España de la primera edición en castellano, 1994, de la primera edición en alemán de 1922.
} 
cultura los usos y las costumbres son fuentes de Derecho, por cuanto la repetición y la asimilación las fija en la vida social. Por eso Weber arguye que las reglas convencionales representan normalmente la manera como se convierten en puras y efectivas regularidades de actuar, meras costumbres, por lo tanto, en normas obligatorias, garantizadas casi siempre por la coacción psíquica. Y el simple hecho de la repetición regular de fenómenos favorece que tales fenómenos adquieran la dignidad de algo normativamente ordenado ${ }^{5}$.

El ordenamiento jurídico influye siempre, en función de su estructura, en la distribución del poder, del que la precedencia pública de quienes lo encarnan es expresión. Como nos enseña Weber "El reconocimiento del poder, tanto político como económico es retribuido como un honor social. El orden jurídico puede garantizar tanto el poder como la existencia del honor [...] Llamamos "orden social" a la forma en que se distribuye el honor social dentro de una comunidad entre grupos típicos pertenecientes a la misma". He ahí una de las funciones del Protocolo.

\subsection{Herramientas para la construcción de la Teoría del Protocolo como Ciencia.}

“El conocimiento científico se caracteriza por ser: analítico, aplicativo, comunicable, crítico, específico, explicativo, objetivo, impersonal, legal, metódico, predictivo y por usar el lenguaje científico. También se caracteriza por ser producto de la investigación, provisional, reflexivo, sintético, sistemático, transformador, universal y verificable" (Terrones, 2009). Esta reflexión resulta de enorme utilidad da la hora de construir una teoría científica que sustente nuestra pretensión de convertir el Protocolo de técnica a ciencia. A través del análisis o la analítica desagregamos el todo en sus elementos o partes, a fin de descubrir su mecanismo interno y sus relaciones, captar su esencia y explicar su movimiento externo. Cuando descubrimos las leyes de la realidad social, las aplicamos. El conocimiento científico debe ser comunicable, entendible y asequible. No se guarda, se divulga, se expande, se comparte. Por ello, la ciencia debe ser explicativa; es decir, explicarse.

\footnotetext{
${ }^{5}$ En el Capítulo I del Código Civil español, donde se especifican las Fuentes del Derecho, se señala:

1. Las fuentes del ordenamiento jurídico español son la ley, la costumbre y los principios generales del derecho. 2. Carecerán de validez las disposiciones que contradigan otra de rango superior. 3 . La costumbre sólo regirá en defecto de ley aplicable, siempre que no sea contraria a la moral o al orden público y que resulte probada. Los usos jurídicos que no sean meramente interpretativos de una declaración de voluntad tendrán la consideración de costumbre. 4. Los principios generales del derecho se aplicarán en defecto de ley o costumbre, sin perjuicio de su carácter informador del ordenamiento jurídico. 5. Las normas jurídicas contenidas en los tratados internacionales no serán de aplicación directa en España en tanto no hayan pasado a formar parte del ordenamiento interno mediante su publicación íntegra en el «Boletín Oficial del Estado».6. La jurisprudencia complementará el ordenamiento jurídico con la doctrina que, de modo reiterado, establezca el Tribunal Supremo al interpretar y aplicar la ley, la costumbre y los principios generales del derecho.7. Los Jueces y Tribunales tienen el deber inexcusable de resolver en todo caso los asuntos de que conozcan, ateniéndose al sistema de fuentes establecido.
} 
El conocimiento científico se adquiere mediante la observación y reflexión crítica, desinteresada y metódica de los hechos y que son comprobados por la experiencia. La ciencia es específica en cada caso. El concepto de Ciencia Universal debe interpretarse como suma de saberes, como Enciclopedia; pero cada ciencia es específica, versa sobre una cosa concreta. La del Protocolo debe ceñirse a su campo. Se dice que es fáctico u objetivo, por cuento parte y regresa a los hechos, tras haberlos analizado. Terrones, 2009), precisa:

Se dice que el conocimiento científico es objetivo porque el pensamiento científico se adapta a la cosa y está fundamentado en hechos que nadie puede negar y con las cuales la inteligencia del hombre de ciencia trabaja con independencia, sin dejarse influir por los instintos, los sentimientos, las pasiones, los deseos e intereses de las personas. El conocimiento científico aspira a eliminar la subjetividad, los elementos afectivos y volitivos (emoción, pasión, sentimiento, ilusión, voluntad, etc.) y a invocar solamente las razones de la razón. Lo que el conocimiento científico enuncia y confirma de los hechos investigados se denomina datos empíricos.

Por último, se sobrepone a la persona, es impersonal, usa un lenguaje preciso, pero que requiere de ciertos fundamentos precios, de convenciones sociales para interpretar las claves de su propia exposición. "El conocimiento científico está organizado e íntimamente ligado, conectado y encadenado de manera lógica entre sí y se apoya en la lógica para la construcción de un sistema de ideas o de sus propias teorías. Un pensamiento sirve de base al que le sigue y así sucesivamente" (Terrones, 2009). Hay dos características especialmente llamativas: que debe ser universal, los conocimientos adquiridos son válidos para todas las personas y que es comprobable, es decir, tiene propiedades que a través de métodos, técnicas y medios científicos permiten establecer el grado concreto de certidumbre y exactitud de los conocimientos.

Frente a la voluntad de crear una ciencia del Protocolo ha aparecido un fenómeno que, aparentando lo mismo, pretende justamente lo contrario: El proceso comercialreduccionista que pretende reinventar, con varias líneas de producto, la profesión de Protocolo, convirtiéndola en un sindicato de actividades diversas, pese al corifeo que lo acompaña no va a ayudar en nada a los fines que dice perseguir. Pero por el camino que vamos parece que quieren convertir al jefe de protocolo en un organizador de bodas y bautizos, como gráficamente dice la doctora Alvarado.

La cuestión radica en la contradicción de que, por un lado, se pretenda articular un sistema de reflexión creativa y crítica, que eleve el Protocolo a la condición de Ciencia, y por otro no se dude en convertir la profesión de quienes han de ejercerla en una especie de ómnibus, que valga para todo, invadiendo sin el menor pudor ámbitos que ya cubren otras carreras y titulaciones del ecosistema de la Comunicación.

La profesión de Protocolo o los profesionales del Protocolo son una realidad solvente, presente, conocida, ejerciente y visible. Y esos profesionales lo son, no por poseer una patente administrativo-académica, sino per se, porque lo confirman cada día con sus actos, con independencia de la formación de origen que es variada: desde La autodidacta a la reglada. 


\subsection{Interferencias y valores en la construcción de la Ciencia del Protocolo.}

No ayuda nada a que la comunidad científica acepte que se puede desarrollar una verdadera ciencia, como se entiende como tal, el estudio analítico del protocolo y sus actividades relacionadas, la desaforada invención que han puesto en marcha determinados sectores privados relacionados con este mundo. Ni todo es ciencia ni todo puede ser considerado ciencia. Un zapatero puede ser un excelente componedor de calzado, pero lo suyo no es una ciencia, sino una habilidad o si se quiere, hasta un arte menor o artesanía...Un organizador de bodas puede ser un excelente gestor de las contratas para estos menesteres, pero eso no tiene ciencia, ni es ni será una ciencia.

Lo más insólito es que, desde planteamientos comerciales y de marketing, se pretende abanderar la formulación de una doctrina, en la que invocan sin rubor términos como universitario y científico. ¿A alguien con sentido común se le puede ocurrir que vaya a desarrollarse una ciencia sobre la organización de bodas, la organización de un concierto de rock o cosas semejantes? A las diferentes ramas de las Ciencias de la Comunicación les cuesta mucho trabajo avanzar y consolidar sus posiciones en el universo del conocimiento. No se pueden considerar, en rigor, científicas lo que no pasan de habilidades más o menos operativas y meramente mecánicas o prácticas. Pero eso no es ciencia ¿Nos imaginamos una tesis doctoral que se titulara: "Epistemología de la boda. El banquete nupcial y sus variantes socioculturales. Del merengue a la nata montada". Otro título "Heurística de los Premios Goya. El largo de falda de las actrices como elemento determinativo de mensaje postmoderno". Y todavía un tercero: "El discurso presidencial en el almuerzo de la junta general de una sociedad por acciones. Prosodia y casuística del balance como género literario".

La nueva ubicación del Protocolo como carrera universitaria, con todos los niveles previstos, obliga a un replanteamiento de su consideración como mera técnica formal al servicio de la imagen y la representación simbólica de las instituciones y los organismos, a través de los actos públicos. Su inclusión entre las carreras tradicionales de la Comunicación, de mayor abolengo y trayectoria, requiere dotarla de líneas de investigación rigurosas que supere la mera casuística meramente descriptiva de ceremonias y ritos, para profundizar en el estudio social, jurídico e histórico de los elementos que la conforman. Al convertirse en una carrera específica debe desarrollar, para serlo, su propio ámbito científico.

Lo científico se entiende, en cada ocasión, según la conveniencia de quien lo define. De modo que corremos el riesgo de considerar como científico algo que no lo es. $\mathrm{O}$ al contrario. ¿Es el protocolo una ciencia como tal? Si tomamos con referencia el mercado bibliográfico, nos asalta la primera duda: existe una interminable serie de monografías al respecto que, o bien tienen un carácter de manual práctico, descriptivo de la casuística más variada, pero que no siempre profundiza en el porqué de sus afirmaciones; o bien se trata de trabajos históricos, donde el relato de episodios del pasado, situaciones anecdóticas o sucesos variados extrae consecuencias sociológicas o políticas que sí elevan el rango de la publicación. 
Podríamos argumentar, en este caso, que el "Protocolo" no tiene fines propios, sino que es un instrumento para mejorar la presentación en público de las instituciones o, si se prefiere, visualizar el poder, la jerarquía y el rango de los personajes y las instituciones públicas. Por lo tanto, no pasaría de ser una técnica, pero sin los requisitos para ser considerado propiamente una ciencia. Pero quienes defienden su vigencia como conocimiento específico replican que lo mismo ocurre con las Relaciones Públicas, la Publicidad o el Periodismo, que carecen de objeto propio por sí mismos, sino que son artes al servicio de otros fines. No es menos cierto que incluso las ciencias más puras son ciencias de la utilidad; es decir, son aplicables a objetos distintos del conocimiento mismo. Dicho de otro modo, sirve para algo a la sociedad.

\section{CONCLUSIONES}

La reflexión intelectual es el camino que convierte una actividad profesional en una ciencia al dotarla, a partir de la experiencia, de los elementos de contraste (y controversia) que la definen y enriquecen. Desde la observación de los fenómenos sociales se formulan hipótesis que van dando lugar a teorías contrastables o tesis, que a su vez van fijando los contenidos de esa nueva ciencia. Ese es el camino siempre. Ese ha de ser el de la ciencia del Protocolo. Para que una práctica profesional se desgaje de la familia intelectual que la aloja es preciso que se desarrolle como ciencia, y eso no se logra solamente porque en el Boletín Oficial del Estado se creen nuevas carreras o la ANECA la santifique, no siempre acorde con sus propios postulados. A veces, las urgencias comerciales son falsos aliados.

Son ponderables todas las iniciativas que hagan avanzar el protocolo como ciencia, corrigiendo la urgencia de algunos pasos anteriores que la experiencia ha revelado imprecisos. Siempre he dicho que, a mi entender, el planteamiento inicial de las carreras de comunicación, cuando éstas se incorporan a la Universidad en 1970, estaba bien trazado: un primer periodo de formación básica común, y un segundo de especialización específica. La aparición de la carrera de Protocolo, separada del núcleo las Relaciones Públicas, debería haber aconsejado un periodo previo de publicaciones, trabajos científicos, estudios y aportaciones que armaran la nueva ciencia más allá de la mera casuística práctica.

Sobre este asunto se lleva reflexionando en la Universidad desde hace muchos años, como lo prueban las tesis doctorales ya aportadas, y que encabeza en número la Universidad de Sevilla. Pero, pese a que es un tema viejo en la agenda diaria de los investigadores que trabajamos en este ámbito en España, se debe subrayar que tiene como marco referente la universidad pública española. Véanse los trabajos de los doctores Dolores del Mar Sánchez, Marta Pulido, María Teresa Otero, Salvador Hernández y otros en este terreno.

- Para convertir al Protocolo en una ciencia solvente dentro del conjunto de la Comunicación, su teoría debe construirse a partir del Derecho, la Sociología, la Historia, la Ética y la Estética, entre otras muchas, emparentada con los ámbitos donde históricamente se ha desempeñado como técnica de ordenamiento y de organización social. 
- Le teoría científica debe ignorar que en el mercado existe una oferta de estudios, carreras, cursos y actividades más o menos regladas absolutamente excesiva, no siempre con la calidad y el contraste necesario. Es una actividad que, desde su perspectiva comercial, se reinventa cada día: se ofrecen nuevas carreras, estudios especialidades o salidas, que son lo mismo de siempre. En la construcción de la nueva ciencia se deben evitar la interferencia de estos sectores mercantiles y comerciales, que tratan de dar forma a la doctrina en función de sus intereses y perspectivas.

- Se debe ignorar que el Protocolo es una cuestión de moda, aislando su estudio de los enfoques meramente publicitarios que perturban el objetivo marcado. Se precisa hallar los puntos de encuentro con otras ciencias de la comunicación y a partir de estas experiencias extraer, aprovechar y desarrollar las propias formulaciones.

\section{REFERENCIAS}

Alcina Franch, José (1999). Aprender a investigar, Madrid: Compañía Literaria.

Ander-Egg, Ezequiel (1996), Técnicas de Investigación social, México: El Ateneo.

Babini, José (1975). El saber. Buenos Aires: Galatea-Nueva Visión.

Cohen, M. y Nagel, E. (1973). Introducción a la lógica y al método científico. Buenos Aires: Editorial Amorrortu.

González Rio, Ma José. (1997), Metodología de la investigación social: Técnicas de recolección de datos, Alicante: Aguaclara.

Hempel, Carl (1982), Filosofía de la ciencia natural, Madrid: Alianza.

Otero Alvarado, Ma Teresa (1999). Protocolo y relaciones públicas de Estado. Los días nacionales en la exposición Universal de Sevilla. Tesis Doctoral. Universidad de Sevilla. Facultad de Comunicación.

Sarres, Jean (1982). Manuel practique de Protocole, París, Editions de la Blevre.

Sgrelli, Massimo (2006). Il Ceremoniale, El Ceremoniale moderno e il Protocolo di Stato. Regolee scritte e non scritte, Roma, Master Edizioni.

Terrones Negrete, Eudoro (2009). El conocimiento científico y su caracterización. (Blog personal. http:/ / eudoroterrones.blogspot.com.es/2009/03/el-conocimiento-cientificoy-su.html. [Recuperada 7 de agosto de 2013]

Weber, Max. (1993) Economía y Sociedad, Madrid, Fondo de Cultura Económica, décima reimpresión para España de la primera edición en castellano, 1944, de la primera edición en alemán de 1922). 


\section{Fernando Ramos Fernández}

Doctor en Ciencias de la Información por la Universidad Complutense de Madrid. Licenciado en Periodismo por la misma Universidad y Técnico en Radiodifusión y Televisión por la Escuela Oficial de Madrid. Profesor Titular de Deontología y Derecho de la Información y la Publicidad en la Universidad de Vigo. Dirige el Foro HispanoLuso Iberoamericano de Protocolo. Imparte el curso de Doctorado en Comunicación institucional y Protocolo en el Master de Lengua y Comunicación en los Negocios. Profesor invitado de diversas universidades españolas y extranjeras en cursos especializados superiores y masters de Comunicación y Protocolo. Director de la Academia de Protocolo de la Organización Internacional de Ceremonial y Protocolo. 